\title{
Expression of matrix metalloproteinase-9 in skin lesions of leprosy patients: the difference between paucibacillary and multibacillary cases
}

\author{
Garima Singh $^{\mathrm{a}}$, Aarzoo Jahan ${ }^{\mathrm{a}}$, Ruchika Gupta ${ }^{\mathrm{b}}$, Navmeet Soin ${ }^{\mathrm{a}}$, \\ Leela Pant ${ }^{\mathrm{a}}$, Namrata Sarin ${ }^{\mathrm{a}} \&$ Sompal Singh ${ }^{\mathrm{a}}$ \\ a Department of Pathology, Hindu Rao Hospital, Delhi, India \\ ${ }^{\mathrm{b}}$ ICMR-National Institute of Cancer Prevention and Research, Noida, India
}

Submitted 4 August 2020; Accepted 16 October 2020

\begin{abstract}
Summary
Background and objective: Mycobacterium leprae causes leprosy, which is a chronic granulomatous disease showing a wide spectrum of skin lesions and typically involves peripheral nerves. The role of the matrix metalloproteinases (MMPs) in the pathogenesis of various clinico-pathological forms of leprosy has not yet been fully explored. Our study had the objective to evaluate the expression of MMP-9 in cutaneous lesions of paucibacillary and multibacillary leprosy patients.

Methods: Skin biopsies from 31 newly diagnosed leprosy patients were assessed immunohistochemically for expression of MMP-9. Depending on the number of skin lesions, the patients were categorized into two groups, where group I comprised paucibacillary leprosy (PB) patients and group II comprised of multibacillary leprosy (MB) patients.

Results: PB leprosy cases (tuberculoid pole) showed moderate to marked MMP-9 expression within the granulomas in and around epithelioid cells while the immunoreactivity was weak in the MB (lepromatous pole) leprosy cases. This difference was statistically significant between the two groups. $(p$ value $=0.001)$.

Conclusion: The varied response to Mycobacterium leprae infection may be attributed to MMP-9, through its role in inflammation, granuloma formation and nerve destruction. Hence, neo-adjuvant treatment in the form of MMP inhibitors may offer benefit in those clinical forms of leprosy with high MMP-9 expression and open new opportunities for therapeutic interventions.
\end{abstract}

Keywords: Hansen's disease, Leprosy, skin lesions, matrix metalloproteinases, MMP-9

Correspondence to: Dr Sompal Singh (MD), Specialist, Department of Pathology, Hindu Rao Hospital, Delhi, India (e-mail: sompal151074@gmail.com) 


\section{Introduction}

Mycobacterium leprae (M. leprae) is an obligate intracellular microbe which causes leprosy, a chronic granulomatous disease. ${ }^{1}$ Till date, no useful vaccines or successful immunotherapeutic tools could be developed against leprosy. Based on the type and the graded immune response of the host to M. leprae, the disease is categorized into six types: Indeterminate (I), Tuberculoid Leprosy (TT), Borderline Tuberculoid (BT), Mid-Borderline (BB), Borderline Lepromatous (BL) and Lepromatous Leprosy (LL). ${ }^{2}$ However, the World Health Organization (WHO) uses the number of skin lesions to categorize leprosy patients into two groups, i.e. paucibacillary (PB) and multibacillary leprosy (MB). ${ }^{3}$

At the tuberculoid pole of this disease, a well-expressed cell-mediated immunity and delayed hypersensitivity effectively controls bacillary multiplication with the formation of epithelioid cell granulomas. Clinically, these patients present with few (usually less than five) hypoesthetic or anesthetic skin lesions along with asymmetrical nerve involvement and the skin biopsy shows granulomas surrounding neurovascular bundles in the papillary zone with no detectable acid fast bacilli (AFB). The lepromatous form, on the other hand, has cellular anergy toward $M$. leprae with resultant abundant bacillary multiplication and inactivated macrophages. ${ }^{4}$ Lepromatous leprosy patients present with numerous symmetrical skin lesions along with symmetrical nerve thickening and glove and stocking anesthesia. 5,6 On histopathology, the lepromatous pole presents numerous foamy macrophages, along with few lymphocytes and plasma cells in the dermis, and numerous AFBs, singly or in clumps (globi). ${ }^{4}$

Matrix metalloproteinase enzymes (MMPs) come under the family of zinc dependent extracellular proteases, which act to break down the extracellular matrix (ECM) components. ${ }^{7}$ MMPs are secreted by stromal and inflammatory cells, as a reactionary response toward exogenous insults or endogenously produced cytokines such as tumor necrosis factor (TNFa) and interferon (IFN- $\gamma$ ) by the inflammatory cells. ${ }^{8}$ This in turn further facilitates leucocyte recruitment, with increased cytokines and chemokine levels, activation of defensin and extracellular matrix remodeling at the site of wound healing. ${ }^{9}$ In the past MMPs were considered to be enzymes specific for matrix degrading, but they have now been proven to have a significant role in immunomodulation, cellular migration and angiogenesis. ${ }^{10-12}$ They are broadly classified according to substrate specificity into collagenases (MMP-1, 8 and 13), gelatinases (MMP-2 and -9), stromelysins (MMP-3, -10, -11), elastases (MMP-7 and -12) and membrane-type MMPs (MT-MMPs; MMP-14, -15, -16 and -17). ${ }^{13}$ The proteolytic activity of MMPs is inhibited by specific tissue inhibitors of metalloproteinase (TIMPs), which exist in complex form with MMPs. The MMP:TIMP ratio is critical for regulation of the proteolysis of connective tissues and for control of tissue damage. ${ }^{10}$

As gelatinases (MMP-9) are known to degrade the basement membrane components, they may act as critical molecules in directing the migration of leucocytes toward the focus of inflammatory reaction and are hence crucial for the containment of infections. ${ }^{14}$

MMP-9 is produced by keratinocytes, monocytes and macrophages. ${ }^{15}$ MMP-9 is partly responsible for T-cell and dendritic cell migration. ${ }^{16}$ Of several MMPs known, till date, MMP9 is regarded as the chief MMP which is secreted by monocytic cells. ${ }^{17}$ During infection with Mycobacterium tuberculosis, MMP-9 is released by infected monocytes and multinucleate giant cells, leading to blood-nerve barrier breakdown in tuberculous meningitis. ${ }^{18,19}$ Several studies have documented a definitive role for MMP-9 in fibrotic interstitial lung diseases and chronic obstructive pulmonary disease..$^{20,21}$ 
Exploration of the functions of MMP-9 in leprosy has been fairly recent. Teles et al. compared mRNA levels of MMP-9 in lesions and serum. They found elevated MMP-9 mRNA levels in tuberculoid lesions as compared to lepromatous lesions. ${ }^{22}$ Another study reported higher levels of serum MMP-9 in PB patients compared with MB patients. ${ }^{23}$

However, the differences in MMP-9 expression in skin lesions of leprosy have not been studied till date. Hence, this study was conducted to shed light on the modulating effect of MMP-9 in the pathogenesis of leprosy.

\section{Materials and methods}

The present study included only newly diagnosed cases of leprosy attending the Dermatology Outpatient Department over a period of 1 year (August 2011 to September 2012). The patients were categorized as PB and MB, based on the count of cutaneous lesions. Patients with more than five skin lesions were classified as MB leprosy and those with five or fewer lesions were categorized as PB leprosy. ${ }^{24}$ Patients not giving consent for inclusion or those with coexisting chronic illness like diabetes, tuberculosis or leishmaniasis were excluded from the study. After gaining written informed consent, a wedge skin biopsy was performed, the biopsy specimen was processed, followed by hematoxylin and eosin staining. Wade-Fite stain was performed to specifically detect $M$. leprae bacilli.

In all cases, immunostaining for MMP-9 was done by using a standard immunoperoxidase method. Antigen retrieval was done using a microwave oven at $\mathrm{pH} 6.0$ provided by citrate buffer. Overnight incubation was done for slides with primary antibody (MMP-9, Biogenex, USA) followed by application of super-enhancing reagent and finally with poly-HRP kit (Biogenex, USA) and DAB as the chromogen. MMP-9 positivity was seen as cytoplasmic immunereactivity with heterogeneous pattern and varied distribution in macrophages, endothelial cells and fibroblasts. The intensity of MMP-9 immunostaining was categorized into the following categories: mild (weak, but detectable staining), moderate (distinct staining), and severe (intense staining). ${ }^{25}$ MMP-9 positivity in keratinocytes was not assessed in this study.

\section{Statistical analysis}

Numerical data were analyzed using the Mann-Whitney test while comparison of categorical data was done through the Chi-square test. $P$ value of $<0.05$ was considered significant. Approval for conducting this study was obtained from the Institutional Ethics Committee.

\section{Results}

The study included 31 newly diagnosed leprosy cases. Of these, 18 (58.06\%) were PB leprosy and 13 (41.94\%) were MB leprosy. The age of subjects ranged from 11 to 70 years with the highest numbers $(25.81 \%)$ in the fifth decade of life. The mean age of PB patients was 32.94 years and MB patients was 41.85 years, with no statistically significant difference between them $(p$-value $=0.081$, Mann-Whitney U Test). A majority of patients $(64.52 \%)$ were males with M: F ratio of 1.8:1. No significant difference was found in the M:F ratio between $\mathrm{PB}$ and MB groups $(p$-value $=0.291$, Pearson Chi-Square Test $)$.

Biopsies from PB leprosy cases demonstrated moderate (5 cases, 27.78\%) to marked (13 cases, $72.22 \%$ ) MMP-9 staining, mainly concentrated within the granulomas in and around epithelioid cells (Figure 1). On the other hand, MB cases showed weak (4 cases, 30.77\%) to moderate (8 cases, $61.54 \%$ ) staining intensity of MMP-9 in histiocytes dispersed in the dermis (Figure 2). Only 1 case (7.69\%) of MB leprosy demonstrated marked staining intensity of 


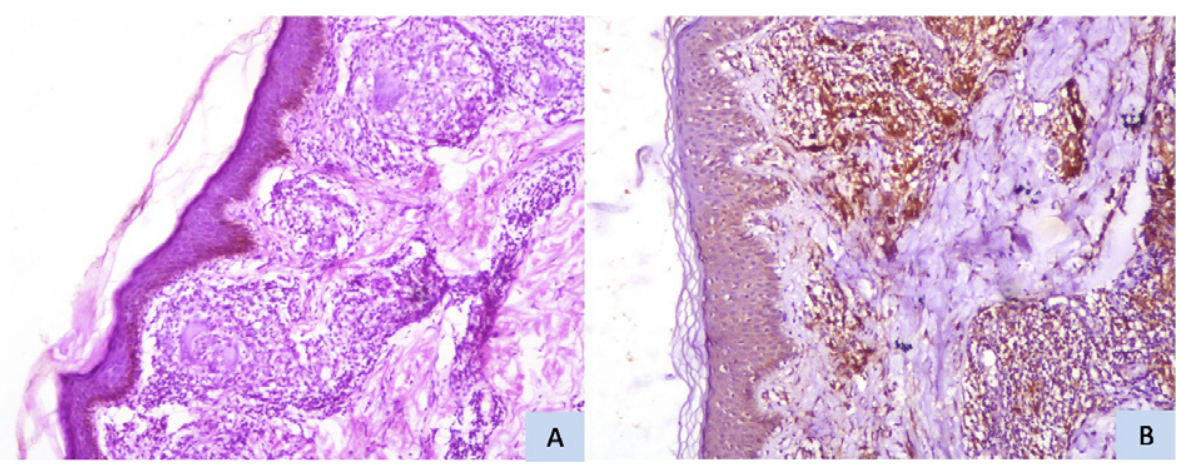

Figure 1 A. Photomicrograph of a paucibacillary case with epithelioid cell granulomas and Langhan giant cells [H\&E, 40X10x]. B. Immunostaining showing intense expression of MMP-9 in epithelioid cells [MMP-9 DAB, 40X10x].

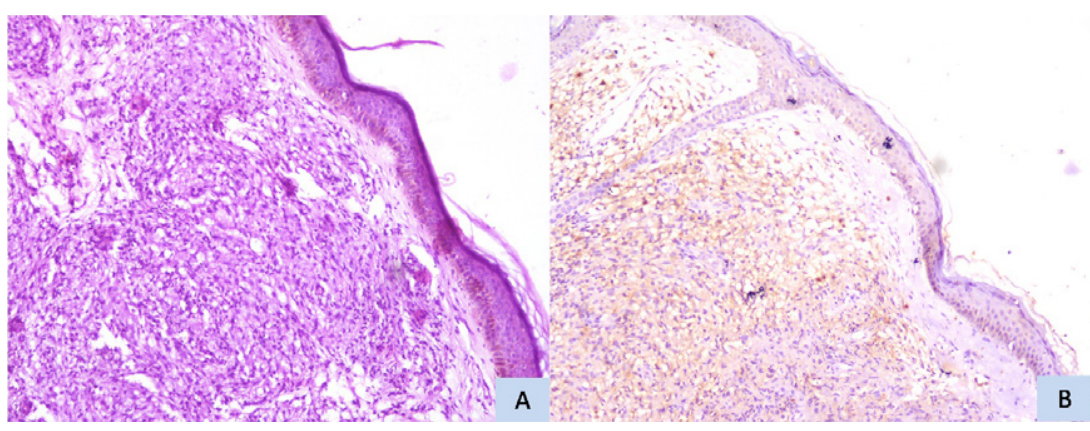

Figure 2 A. Photomicrograph of a multibacillary case with groups of foamy cells in the dermis [H\&E, 40X10x]. B. Immunostaining showing mild-moderate expression of MMP-9 in foamy cells [MMP-9 DAB, 40X10x].

MMP-9, in histiocytes dispersed in the dermis. A statistically significant difference was found in the intensity of staining with MMP-9, between PB and MB leprosy ( $p$-value $=0.001$, Pearson Chi-Square Test, Table 1, Figure 3).

\section{Discussion}

Leprosy is a chronic disease having a wide array of clinical presentation and pathological subtypes, determined by the cell-mediated immune response of the host towards M. leprae. On one end of the spectrum is tuberculoid leprosy characterized by the formation of organized epithelioid cell granulomas, as a result of controlled bacillary multiplication due to efficient cell-mediated immunity. These patients have marked lymphocytic proliferation (in vitro) and positive skin tests (Mitsuda reaction). At the other end of the spectrum, lepromatous leprosy lacks a cellular response and permits abundant bacillary multiplication. It is characterized by non-activated macrophages, reduced or absent lymphocytic proliferation and non-reactive skin tests. $4,26,27$

As a general rule, whenever the immune system counters an infection, it responds by utilizing MMPs for regulating critical steps such as controlling the movement of effector cells, eliminating the pathogen, resolving inflammation and ultimately leading to remodeling of 


\section{MMP-9 in PB and MB Cases}

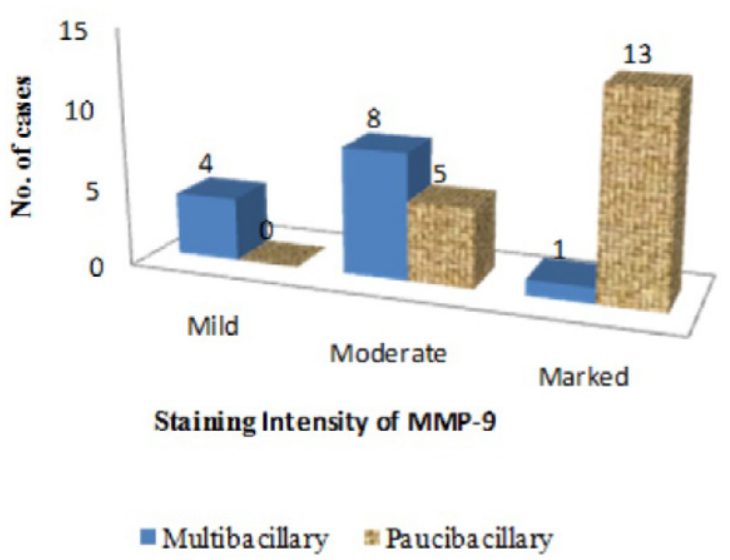

Figure 3 Bar graph showing MMP-9 intensity in multibacillary and paucibacillary leprosy patients.

Table 1. MMP-9 staining intensity in leprosy patients

\begin{tabular}{lccc}
\hline & \multicolumn{3}{c}{ MMP-9 } \\
\cline { 2 - 4 } & Mild & Moderate & Marked \\
\hline Paucibacillary group $(n=18)$ & $0(0 \%)$ & $5(27.78 \%)$ & $13(72.22 \%)$ \\
Multibacillary group $(n=13)$ & $4(30.77 \%)$ & $8(61.54 \%)$ & $1(7.69 \%)$ \\
Total & 4 & 13 & 14 \\
\hline
\end{tabular}

(Pearson Chi-Square Test, $p$-value $=0.001)$.

the extra-cellular matrix. MMPs contribute by aiding in concentrated leucocyte recruitment, increasing cytokine and chemokine production and activation of defensin at targeted sites. ${ }^{15}$ Thus, a suitably titrated action of MMPs can result in the desired effective immune response. On the other hand, an overly exaggerated MMP enzymatic response can be counter productive to the host. ${ }^{13}$ In an unchecked secretory environment, the MMPs may themselves destroy their antagonist protease inhibitor enzymes such as $\boldsymbol{a}$-1 antitrypsin. This results in excessive and unrestricted breakdown of the extra-cellular matrix, which causes an adverse outcome to the local microenvironment of the cell. ${ }^{28,29}$

T-cell and dendritic cell migration has been established to be, in part, MMP-9 dependent. ${ }^{12}$ MMP-9 also plays an important role in fibrosis in end-stage neuritic leprosy. ${ }^{30}$ Fibrosis, in turn, impairs nerve fiber regeneration, making the nerve damage in leprosy irreversible even after the infection has been cured. One study carried out immunoreactive profiling of MMPs in subjects with pure neuritic leprosy (PNL). There was exceptionally intense immunoreactivity seen amongst macrophages, endothelial cells, Schwann and perineural cells, and in the myelin sheaths of axons in the biopsy studied, thus suggesting a role of MMPs in the pathogenesis of neuropathy in pure neuritic leprosy patients. The authors also showed increased MMP-9 expression in the epithelioid cells in tuberculoid patients and reduced expression toward the lepromatous pole $(p$-value $<0.001) .{ }^{31}$ It may possibly be stated, therefore, that MMP-9 could be used as an immunomarker to label activated macrophages in epithelioid cell granulomas and may be helpful in identifying peripheral nerve damage in PB leprosy patients. 
In our study, we provide further evidence of MMP-9 involvement in the pathogenesis of different clinical forms of leprosy. In PB lesions, moderate to marked staining intensity was concentrated in the cellular components of epithelioid cell granuloma. On the other hand, MB lesions showed weak staining intensity in the histiocytes dispersed in the dermis. Our findings support the hypothesis that MMP-9 is a marker of active CMI in patients with leprosy. Similar results were shown by Youssef et al, in which intense MMP-9 immunoreactivity was seen in epithelioid cells of granulomas of TT and BT biopsies from newly diagnosed untreated leprosy patients compared to mild to moderate immune-reactivity in multibacillary patients. ${ }^{30}$

It has been observed that compared to MB patients, PB patients exhibit higher serum MMP9 levels. ${ }^{23}$ In a study that evaluated cutaneous lesions in lepromatous leprosy and tuberculoid leprosy for levels of mRNA of MMP-9, MMP-2, IFN- $Y$ and TNF- $a$, both in lesions and serum of patients, higher tissue and serum values were reported in tuberculoid leprosy compared with lepromatous patients; a high MMP/TIMP-1 ratio was also demonstrated in the inflammatory states of leprosy, which points to potential mechanisms for tissue damage. ${ }^{22}$ These findings may be useful in the development of new therapeutic drugs and modalities for management of leprosy reactions.

Targeted MMP inhibition is being enthusiastically studied as a new and more precise treatment option, not only for cancer patients but also for inflammatory conditions. ${ }^{18,32-34}$ In view of the possible role of MMP-9 in leprosy, targeted anti-MMP-9 therapy could reduce the disabling potential of this disease and improve the work and social life of the patient. Synthetic inhibitors of MMPs such as BAY 12-9566 that selectively inhibits MMP2, MMP-3 and MMP-9 or MMI270, which is a potent broad spectrum orally active MMP inhibitor inhibiting the gelatinases (MMP-2 and MMP-9) and stromelysin (MMP-3) have been investigated in tumors such as murine melanoma and human colon tumor xenografts. ${ }^{32,33}$ MMP inhibitors such as GM6001 have a protective role to play in some conditions such as caspase-mediated apoptosis, Myelin basic protein (MBP) degradation due to injury, spinal nerve infiltration by macrophages and inhibited astrocyte activation in the spinal cord. ${ }^{35}$

One limitation of the present study was the small sample size. Studies with larger number of patients are required to substantiate and validate our findings.

\section{Conclusion}

Our study provides evidence of a role of MMP-9 expression in the degree of inflammation and granuloma formation in various forms of leprosy. New adjuvant treatment in the form of specific or broad-spectrum MMP inhibitors may offer benefit for various clinical presentations of leprosy with improvement in the daily life of patients.

\section{Authors Contributions}

Dr Garima Singh: Literature search, writing up the manuscript

Dr Aarzoo Jahan: Literature search, Proof reading

Dr Ruchika Gupta: Literature search, Proof reading

Dr Navmeet Soin: Help in IHC, Literature search

Dr Leela Pant: Literature search, Head of Department

Dr Namrata Sarin: Guidance in writing manuscript

Dr Sompal Singh: Concept, Data Analysis, Guidance in writing manuscript. 


\section{Conflict of Interest}

There is no conflict of interest.

\section{References}

1 Harboe M. Overview of host-parasite relation. In: Hastings RC, Opromolla DVA (eds), Leprosy. 2nd edn, Edinburgh: Churchill Livingstone, 1994; pp. 87-112.

2 Ridley DS, Jopling WH. Classification of leprosy according to immunity: A five-group system. Int J Lepr Other Mycobact Dis, 1966; 34: 255-273.

3 World Health Organisation. Technical report series. No.768, 1988 (WHO Expert Committee on Leprosy, 6th report).

4 Lever WF, Lever GS. Histopathology of Skin. 9th edn, Philadelelphia: JB Lippincott Co, 2005; pp. 569-570.

5 Gelber RH. Leprosy (Hansen's disease). In: Harrison's Principles of Internal Medicine. 18th edn, New York: McGraw Hill, Inc, 2011; pp. 1359-1366.

6 Khanna N. Infections. In: Illustrative Synopsis of Dermatology and Sexually Transmitted Diseases. 2nd edn, New Delhi: Modern Publishers, 2007; pp. 187-229.

7 Vihinen P, Alaaho R, Kahari VM. Matrix metalloproteinases as therapeutic targets in cancer. Curr Cancer Drug Targets, 2005; 5: 203-220.

8 Unemori EN, Hibbs MS, Amento EP. Constitutive expression of a 92-kD gelatinase (type V collagenase) by rheumatoid synovial fibroblasts and its induction in normal human fibroblasts by inflammatory cytokines. $J$ Clin Invest, $1991 ; 88$ : 1656-1662.

9 Visse R, Nagase H. Matrix metalloproteinases and tissue inhibitors of metalloproteinases: Structure, function and biochemistry. Circ Res, 2003; 92: 827-839.

10 Mott JD, Werb Z. Regulation of matrix biology by matrix metalloproteinases. Curr Opin Cell Biol, 2004; 16: $558-564$.

11 Roy R, Zhang B, Moses MA. Making the cut: Protease-mediated regulation of angiogenesis. Exp Cell Res, 2006; 312: $608-622$.

12 Xue M, Le NT, Jackson CJ. Targeting matrix metalloproteases to improve cutaneous wound healing. Expert Opinther Targets, 2006; 10: 143-155.

13 Elkington PT, O'Kane CM, Friedland JS. The paradox of matrix metalloproteinases in infectious disease. Clin Exp Immunol, 2005; 142: 12-20.

14 Okamoto T, Akuta T, Tamura F, Vliet AD, Akaike T. Molecular mechanism for activation and regulation of matrix metalloproteinases during bacterial infections and respiratory inflammation. Biol Chem, 2004; 385: 9971006.

15 Birkedal Hansen H, Moore WG, Bodden MK, Windsor LJ, Birkedal Hansen B, DeCarlo A et al. Matrix metalloproteinases: A review. Crit Rev Oral Biol Med, 1993; 4: 197-250.

16 Xia M, Leppert D, Hauser SL, Sreedharan SP, Nelson PJ, Krensky AM et al. Stimulus specificity of matrix metalloproteinase dependence of human $\mathrm{T}$ cell migration through a model basement membrane. J Immunol, 1996; 156: 160-167.

17 Keeling J, Herrera GA. Human matrix metalloproteinases: characteristics and pathologic role in altering mesangial homeostasis. Microsc Res Tech, 2008; 71: 371-379.

18 Zhu XW, Price NM, Gilman RH, Recarvarren S, Friedland JS. Multinucleate giant cells release functionally unopposed matrix metalloproteinase-9 in vitro and in vivo. J Infect Dis, 2007; 196: 1076-1079.

19 Friedland JS, Shaw TC, Price NM, Dayer JM. Differential regulation of MMP-1/9 and TIMP-1 secretion in human monocytic cells in response to Mycobacterium tuberculosis. Matrix Biol, 2002; 21: 103-110.

20 Hautamaki RD, Kobayashi DK, Senior RM, Shapiro SD. Requirement for macrophage elastase for cigarette smoke-induced emphysema in mice. Science, 1997; 277: 2002-2004.

21 Corbel M, Lagente V, Boichot E. Pulmonary inflammation and tissue remodelling: Role of metalloproteinases. Eur Respir Rev, 2000; 10: 260-263.

22 Teles RM, Teles RB, Amadeu TP, Moura DF, Mendonça-Lima L, Ferreira H et al. High matrix metalloproteinase production correlates with immune activation and leukocyte migration in leprosy reactional lesions. Infect Immun, 2010; 78: 1012-1021.

23 Fitriyanti, Yanwirasti, Jamsari, Pamudji R. The role of immunoglobulin M anti-phenolic glycolipid-1 and matrix metalloproteinase-9 levels in blood serum in peripheral nerve damage of multibacillary leprosy. Drug Invention Today, 2019; 11(10): 2452-2457.

24 WHO, Chemotherapy of leprosy. Technical report series 847, 1994 World Health Organization, Geneva.

25 Lockwood CJ, Oner C, Uz YH, Kayisli UA, Huang SJ, Buchwalder LF et al. Matrix metalloproteinase 9 (MMP9 expression in preeclamptic decidua and MMP9 induction by tumor necrosis factor alpha and interleukin 1 beta in human first trimester decidual cells. Biol Reprod, 2008; 78(6): 1064-1072. 
26 Ramos T, Quintana IZ, Appelberg R, Sarno EN, Silva MT. T-Helper cell sub populations and the immune spectrum of leprosy. Int J Lepr, 1989; 57: 73-81.

27 Bloom BR, Mehra V. Immunological unresponsiveness in leprosy. Immunol Rev, 1984; 80: 5.

28 Desrochers PE, Jeffrey JJ, Weiss SJ. Interstitial collagenase (matrix metalloproteinase-1) expresses serpinase activity. J Clin Invest, 1991; 87: 2258-2265.

29 Liu Z, Zhou X, Shapiro SD, Shipley JM, Twining SS, Diaz LA et al. The serpin alpha1 proteinase inhibitor is a critical substrate for gelatinase B/MMP-9 in vivo. Cell, 2000; 102: 647-655.

30 Youssef SS, Attia EAS, Awad NM, Mohamed GF. Expression of Matrix Metalloproteinases (MMP-3 and MMP9) in Skin Lesions of Leprosy Patients. J Egypt Women Dermatol Soc, 2009; 6: 80-87.

31 Teles RMB, Antunes SLG, Jardim MR, Oliveira AL, Nery JA, Sales AM et al. Expression of metalloproteinases (MMP-2, MMP-9, and TACE) and TNF- $a$ in the nerves of leprosy patients. J Periph Nerv Syst, 2007; 12: 195204.

32 Bloomston M, Zervos EE, Rosemurgy AS. Matrix metalloproteinases and their role in pancreatic cancer: a review of preclinical studies and clinical trials. Ann Surg Oncol, 2002; 9: 668-674.

33 Wood JM, Schnell C, Cozens RM, O’Reilly T, Cox D, Ganu V et al. CGS 27023A, a potent and orally active matrix metalloprotease inhibitor with antitumor activity. Proc Am Assoc Cancer Res, 1998; 39: 83.

34 Matsumoto T, Kumagai J, Hasegawa M, Tamaki M, Aoyagi M, Ohno K et al. Significant increase in the expression of matrix metalloproteinase 7 in primary CNS lymphoma. Neuropathology, 2008; 28: 277-285.

35 Kobayashi H, Chattopadhyay S, Kato K, Dolkas J, Kikuchi S, Myers RR et al. MMPs initiate Schwann cellmediated MBP degradation and mechanical nociception after nerve damage. Mol Cell Neurosci, 2008; 39(4): $619-627$. 